\title{
Circuit
}

Musiques contemporaines

\section{Ticket pour la liberté. Entretien avec Otto Joachim}

\section{Anne Marie Messier}

Volume 19, numéro 3, 2009

Pionniers canadiens de la lutherie électronique

URI : https://id.erudit.org/iderudit/038256ar

DOI : https://doi.org/10.7202/038256ar

Aller au sommaire du numéro

Éditeur(s)

Les Presses de l'Université de Montréal

ISSN

1183-1693 (imprimé)

1488-9692 (numérique)

Découvrir la revue

Citer cet article

Messier, A. M. (2009). Ticket pour la liberté. Entretien avec Otto Joachim. Circuit, 19(3), 38-47. https://doi.org/10.7202/038256ar

\section{Résumé de l'article}

Compositeur aux talents multiples, Otto Joachim a été un des pionniers de la musique électroacoustique au Canada. Lors d'entrevues réalisées en 1997 et en 2009, il raconte sa passion pour la technologie, la création de son studio personnel, ses premières expériences de composition électronique, et il livre ses opinions tranchées sur la musique électronique. On y apprend les détails entourant la création de Katimavik, produite pour le pavillon canadien à l'Expo 67 et première oeuvre canadienne entièrement électronique. Puis, avec une franchise polémique, cet artiste de 98 ans discute des limites du genre en interrogeant la valeur des oeuvres de musique électroacoustique. 


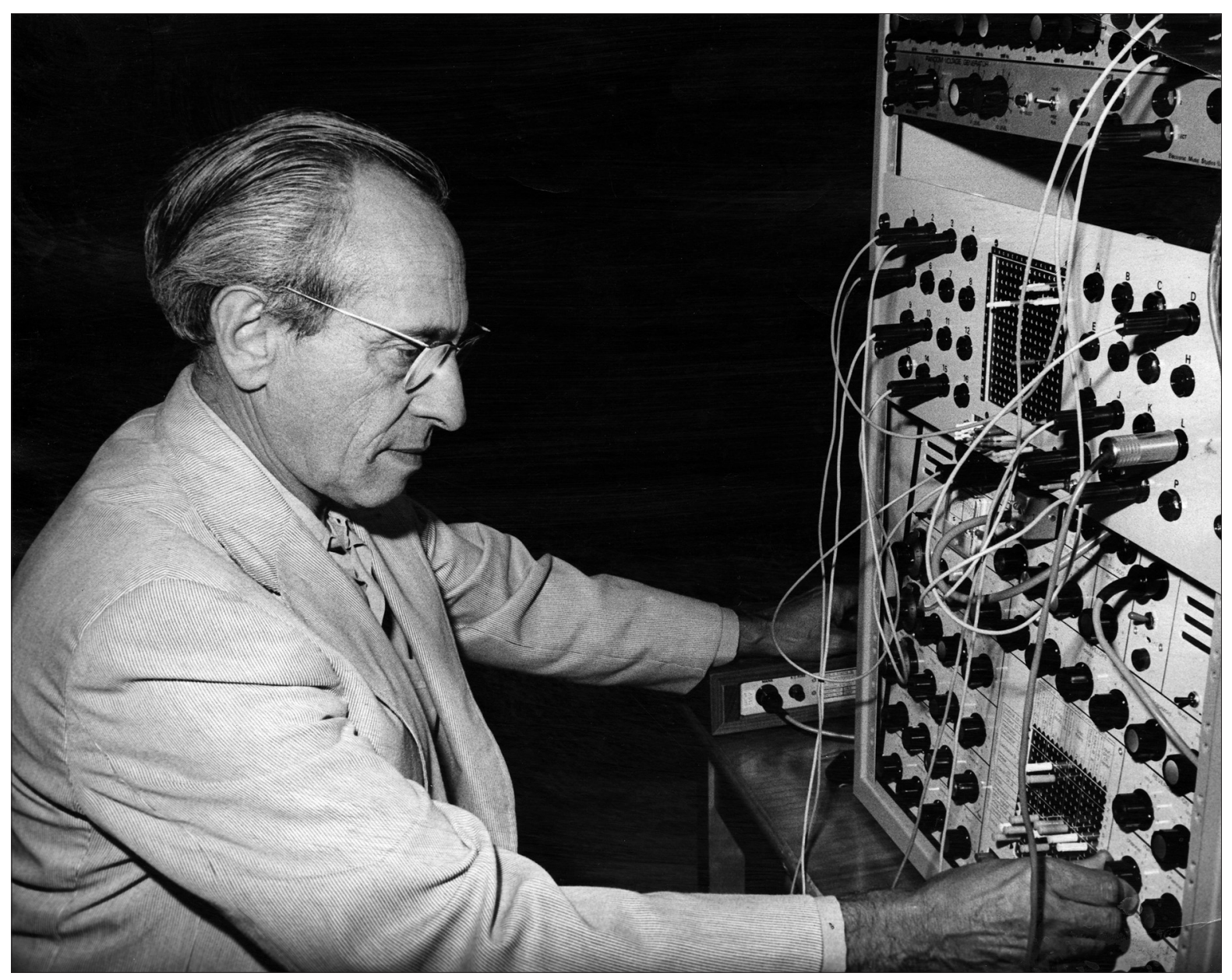

Otto Joachim travaillant sur la partie électronique de Uraufführung, 17 septembre 1973.

(c) Photo Menton, Fonds Otto Joachim, Bibliothèque et Archives Canada, Mus 270/G3. Reproduit avec la permission du donateur. 


\section{Ticket pour la liberté}

\section{Entretien avec Otto Joachim}

Anne Marie Messier

Pour certains compositeurs, la liberté est un impératif; liberté d'expérimentation, liberté de pensée, liberté d’opinion. Avec les menaces nazies, puis japonaises, et enfin américaines sur sa liberté, Otto Joachim, né en 1910, a protégé cette liberté à l'aide d'un talent protéiforme qui lui a permis de s'adapter continuellement aux différents milieux où les circonstances l'ont porté. De son Düsseldorf natal quitté en hâte en 1934 pour Singapour, puis à Shanghai en 1940 peu après la déclaration de la guerre, et enfin à son arrivée à Montréal en 1949, il a d'abord fait une carrière de musicien professionnel; violoniste, chef d'orchestre, altiste à l'Orchestre symphonique de Montréal (1952-1956), fondateur du Quatuor à cordes de Montréal (1955-1963) et du Montreal Consort of Ancient Instruments (1958-1969) pour lequel il a construit des répliques d'instruments $^{1}$. Professeur au Conservatoire de Montréal (1956-1976) et au McGill Conservatorium (1956-1964), il a enseigné le violon, l'alto et la musique de chambre. Très tôt dans sa vie, il a conçu et construit des instruments électroniques de même que de l'équipement électroacoustique. Son corpus d'œuvres illustre son appétit d'expérimentation tout en restant fidèle à la tradition allemande: importance des structures, attachement à la série dodécaphonique, intégration de l'électronique, introduction de l'aléatoire. Sa première œuvre électronique majeure a été Katimavik (1967), diffusée en permanence dans le pavillon canadien à Expo 67. Le texte qui suit est un collage croisé tiré d'abord d'une série d'entrevues menées au printemps 1997 puis d'un nouvel entretien fait en janvier $2009^{2}$.
1. Cinq de ces instruments se trouvent au Musée canadien des civilisations.

2. Les entrevues se sont déroulées principalement en anglais. La traduction est de l'auteure. 


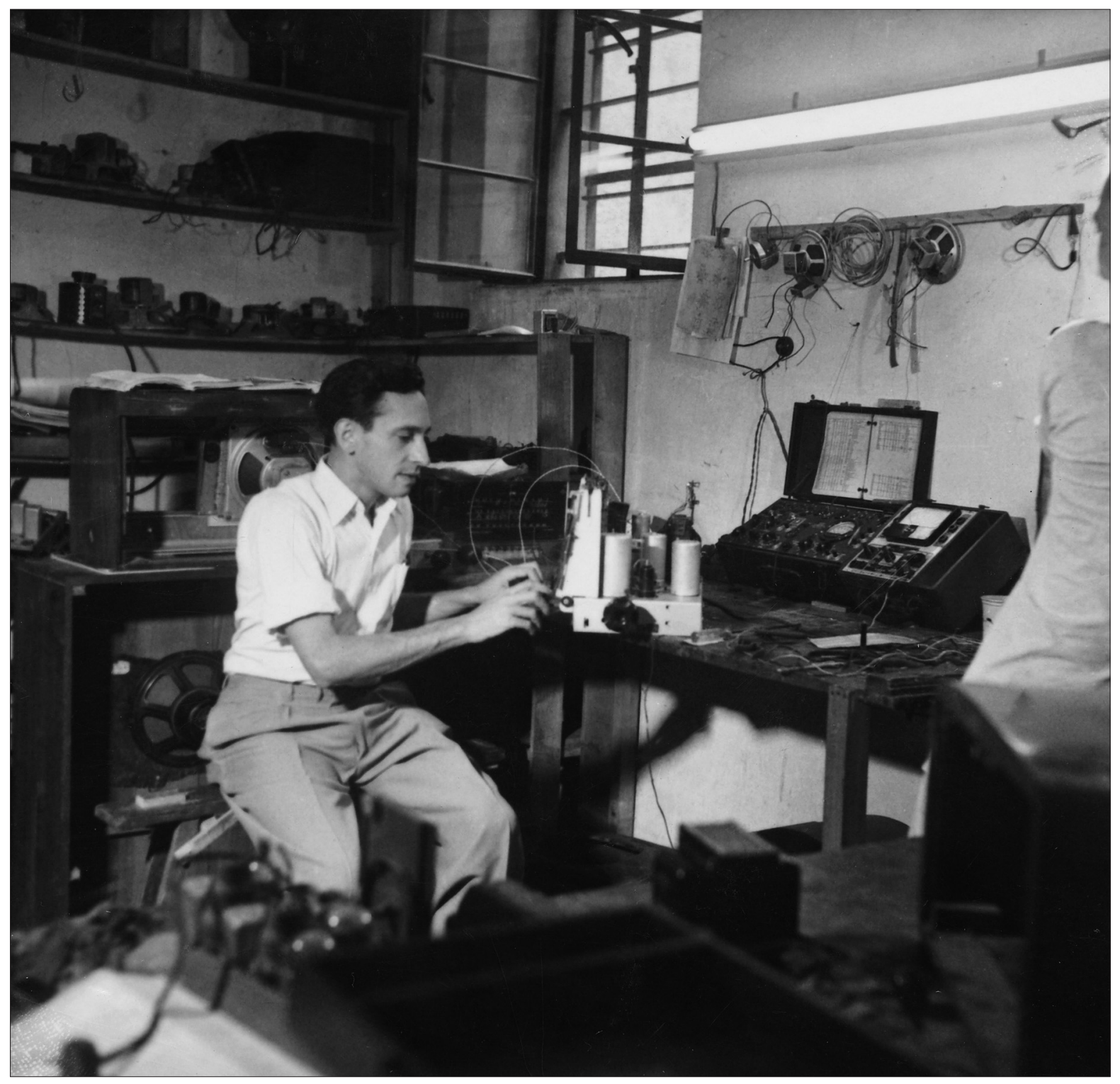

Otto Joachim dans l'atelier de Itkis \& Joachim, Shanghai, circa 1948.

Fonds Otto Joachim, Bibliothèque et Archives Canada, Mus 270/G2. Reproduit avec la permission du donateur. 
2009: AM.M. - Otto, dans les années cinquante, vous avez commencé à acquérir de l'équipement électronique. D'où vous est venu cet intérêt?

0.J. - En fait, cela remonte à 1922, à Düsseldorf. J'avais 12 ans, je m’étais construit un récepteur radio avec deux tubes³ et un châssis de bakélite, plutôt que de métal. Je devais faire très attention, car en approchant ma main, j’obtenais un sifflement. J'ai alors réalisé que je pouvais de cette façon contrôler les sons: je pouvais jouer à cela des heures durant! Bien sûr, c'était à piles puisqu'il n'y avait pas l'électricité. Je me disais qu'un jour, j’allais trouver ou inventer un instrument basé sur ce principe. Plus tard, je me suis acheté un thérémine ${ }^{4}$ et cela a été le tout début de mon intérêt à produire des sons sans instrument acoustique. Bien sûr, entre mes études musicales et mon arrivée à Montréal, il y a eu ces vingt étranges années en Malaisie ${ }^{5}$ et en Chine ${ }^{6}$ pendant lesquelles il ne m’a pas vraiment été possible d'avoir un studio. Pour commencer un studio, encore fallait-il que j’aie une maison où je pouvais faire ce que je voulais! Puis j’ai commencé dans les années cinquante avec d'intéressantes expériences de musique concrète pour un cinéaste d'origine hollandaise dont j’ai oublié le nom ${ }^{7} .$.

1997: AM.M. - Pourtant, vous n'étiez alors pas connu comme compositeur? 0.J. - Non, en fait à cette époque, je n’étais pas compositeur. Même si j'avais écrit de la musique avant, je ne me considérais pas compositeur. Ce n'est qu'en 1953 ou 1954 que je suis vraiment devenu obsédé par l'idée de faire autre chose que de jouer la musique des autres. Je voulais la créer moimême et comme je m’étais enfin établi à Montréal ${ }^{8}$, il n’y avait plus rien pour m’empêcher de le faire. Pour en revenir à cette musique de film, je me suis enregistré jouant sul ponticello sur la corde grave de l'alto, puis j’ai ralenti l'enregistrement d'une octave, puis de deux et enfin je l'ai passé dans une unité de réverbération. Le son que j'arrivais à tirer de cette machine plutôt primitive était formidable. Je savais déjà qu'en ayant joué sul ponticello, il y avait non seulement les sons très élevés, mais aussi les résultantes de la série harmonique et des sons que je ne pouvais entendre, mais que le micro captait. J'avais un type de microphone avec amplificateur qui pouvait capter au-delà de 20000 cycles, et même jusqu'à 40000 cycles. J'ai ensuite manipulé ce son et l'ai utilisé pour ce film. À ma connaissance, rien de ce genre n’avait été fait auparavant.

1997: AMM — D'où venait l'équipement? Du studio de McGill?

0.J. - Je n'ai jamais utilisé le studio de quelqu'un d'autre! J'ai tout fait moi-même. Dès le début des années cinquante, j’ai acheté de l'équipement provenant de surplus de l'armée, en particulier des magnétophones très
3. Le tube électronique avait été inventé quelques années auparavant, en 1904.

4. Inventé par Léon Theremin en 1919, le thérémine est basé sur un oscillateur hétérodyne à tubes électroniques. <http://fr.wikipedia.org/ wiki/Thérémine>, consulté le 15 février 2009).

5. Fuyant l'Allemagne nazie, Otto Joachim a vécu à Singapour de 1934 à 1940, principalement au Raffles Hotel où il était musicien.

6. Son arrivée à Shanghai comme réfugié politique date d'août 1940. Il y a vécu dans le quartier français, ouvrant un magasin d'électronique et de réparation d'instruments pour gagner sa vie. Il y a fabriqué des instruments à amplification électronique ainsi qu'un piano sans table de résonance, à amplification par capteurs électrostatiques.

7. Il s'agit d'Anton Van de Water (PaysBas, 1917 - Québec, 2008).

8. Après la fin de la guerre, Otto Joachim a tenté sans succès d'émigrer aux États-Unis, plusieurs fois refusé pour une question de sécurité nationale jamais élucidée. Ayant ensuite obtenu un visa d'immigration pour le Brésil, il a quitté la Chine en 1949 via le Canada. Le hasard l'a mené à Montréal où il a choisi de rester. 
9. Gustav Ciamaga (Ontario, 1930), compositeur et professeur, a été nommé directeur du Studio de musique électroacoustique en 1965.

10. Kelsey Jones (États-Unis, 1922 - Québec, 2004), compositeur, claveciniste, pianiste et professeur à l'Université McGill.

11. István Anhalt (Hongrie, 1919), compositeur et professeur, s'est établi à Montréal en 1949. Il a mis sur pied le Studio de musique électroacoustique de McGill, qu'il a dirigé de 1964 à 1971. (Cf. entretien dans ce numéro) professionnels. Je suis allé à New York, au célèbre surplus de l'armée américaine sur Canal Street. J'y ai acheté toutes sortes d'équipement sonore. Je ne sais pas à quoi pouvaient bien servir des oscillateurs dans l'armée, mais je m'y suis procuré des oscillateurs à signal carré, des générateurs de pulsation, des générateurs sinusoïdaux... Je voulais m'éloigner de l'école de Cologne qui utilisait l'onde sinusoïdale comme fondamentale en additionnant certaines harmoniques pour créer de nouveaux sons. Pour ma part, je partais d'un son riche en harmoniques puis je le filtrais, ce qui en faisait un procédé plus facile et plus économique et c'est pourquoi au fil des ans, j'ai acquis de nombreux oscillateurs. Je me suis construit une chambre d'écho à neuf têtes en série, et plusieurs autres pièces d'équipement. J'ai aussi créé, un peu plus tard, un appareil pour obtenir des sons transitoires riches, comme une attaque à la flûte avec le son craché, ou le clapet d'un tuyau d'orgue ou le bruit du crin sur une corde avant le son lui-même. Ce qui, en fait, caractérise le son de la flûte, de l'orgue ou du violon! Cela manquait dans la musique produite électroniquement: la coupure du ruban en diagonale ne produisait qu'un crescendo ou un decrescendo. J'ai donc dû inventer des manières d'obtenir une transitoire dans le son; j'ai pris des condensateurs électroniques, les ai chargés avec 300-400 volts puis les ai déchargés sur des oscillateurs. C'était certainement une manière crue de faire les choses, mais cela m'a été extrêmement utile au moment de faire la musique pour le pavillon canadien. Cela donne... vous savez, ce son si intéressant juste avant d'entendre la corne de brume d'un bateau? D'ailleurs, ce son transitoire est aussi très proche du soufflet d'un tuyau d'orgue de 16 ou de 32 pieds. Au XIX' siècle, on a beaucoup cherché à se débarrasser de ce son qu'on trouvait horrible et aujourd'hui on tente de le retrouver...

2009: AM.M. - À ce moment-là, étiez-vous au courant des studios qui se créaient ailleurs?

0.J. - Je savais ce qui se passait à Cologne. J'étais aussi au courant des essais à la Columbia University et à Paris. Mais j'étais très peu informé des activités à Toronto qui fonctionnait plus ou moins en circuit fermé. Gustav Ciamaga9 n'était pas encore là et l'accès au studio y était strictement interdit aux intrus comme moi.

2009: AM.M. - Votre studio a été l'un des premiers au Canada...

0.J. - Le premier studio privé! Il y avait un studio à McGill. Kelsey Jones' ${ }^{10}$ et moi y avions auparavant tenté d'en mettre un sur pied, mais István Anhalt ${ }^{11}$ s'est emparé du projet à un point tel que Jones et moi-même avons perdu tout intérêt. J'ai aussi participé plus tard à la création du studio au Conservatoire. 
Mon studio de l'époque, que je ne qualifierais pas de primitif, était un studio quatre pistes. En fait, il est devenu quatre pistes après avoir reçu la commande pour Expo 67.

1997: AM.M. - Il n'y a rien eu entre ce premier essai pour le film en 1955 et Katimavik en 1967 ?

0.J. - Rien, en effet. Peut-être y avait-il eu une baisse d'enthousiasme ou une diminution de mon pouvoir d'achat... n'oubliez pas que tout ce que je faisais à côté de mon travail de violoniste ou d'altiste était souvent assez mal perçu. Quand je faisais de la musique électronique, les musiciens m'accusaient de les priver de travail! L'encouragement est venu plutôt du côté électronique: par exemple, de Gustav Ciamaga, qui s'est retrouvé plus tard à la tête du studio à Toronto. Avec lui, même si nous avions été tous les deux formés musicalement de manière traditionnelle, nous ne parlions que d'électronique: toute notre correspondance porte sur la manière de construire tel type de générateur, ou comment produire tel son... Ou encore de Kenneth Albert, un technicien de la CBC tout aussi enthousiaste que moi et avec qui je pouvais discuter pendant des heures des possibilités de créer et de manipuler des sons. Alors oui, il y a eu un trou, une pause avant Katimavik, mais mon intérêt a toujours été présent; depuis l'Allemagne de mon enfance à Shanghai où j'ai construit tous ces instruments électroniques, malgré l'occupation japonaise et la difficulté de trouver les fonds nécessaires! Je me rappelle même que construire des instruments passait avant la nourriture... Pour chaque chose commencée en Allemagne, c'est ici que j’ai enfin pu réaliser - je ne dirai pas mes rêves, mais - mes projets. C'est ici que je suis arrivé à fermer la boucle, à faire tout ce que j'avais toujours voulu faire. Ça, c'est Montréal pour moi.

1997: AM.M. - Quand la commande pour le pavillon du Canada de l'Expo 67 est venue en 1965, avez-vous été surpris?

0.J. - Je n'ai jamais vraiment su qui avait pensé à moi. J'ai reçu une lettre qui m'invitait à soumettre une proposition ${ }^{12}$. Le défi était important: non seulement le pavillon était-il immense, mais à ciel ouvert! Il fallait penser à la pluie, au vent qui changeait le son provenant des huit énormes haut-parleurs distribués dans Katimavik. J'ai mis dans ma proposition toute la technique que je pensais nécessaire : les haut-parleurs, les magnétophones. Je ne pouvais leur parler de contenu, il n'y a pas de notation pour la musique électronique!

1997: AM.M. - Vous avez présenté le projet de vive voix?

0.J. - Oh, oui... Il y avait cette énorme table avec peut-être trente personnes inconnues. J'ai su par la suite que Hugh Le Caine'3 était là, mais je ne le connaissais pas encore. J'ai dû leur expliquer tout le matériel que je voulais
12. Lettre du Bureau du Commissaire général signée par Stan White, Chef concepteur, 24 mars 1965.

13. Hugh Le Caine (Ontario, Canada, 1914-1977), concepteur d'instruments électroniques et compositeur. Voir extraits de sa biographie dans ce numéro. 
utiliser, comment il résisterait à six mois de plein air et comment la musique serait en interaction avec les sculptures prévues autour du pavillon. J'ai dû en dire assez puisqu'ils m'ont confié la commande. J'ai attendu un bon moment avant de recevoir les formidables magnétophones demi-pouce à quatre pistes de chez Levis \& Rich en Angleterre, et les ordinateurs pour démarrer et rembobiner automatiquement les bandes. Les magnétophones valaient à eux seuls $10000 \$$ chacun! J'ai commencé à composer piste par piste. La procédure s'est révélée assez difficile. Je me servais des oscillateurs de l'armée, mon invention de sons transitoires, et, bien sûr, de nombreuses unités d'écho et de réverbération. Je composais tout ça ici, chez moi, entouré de haut-parleurs de quinze pouces à chaque coin. Je nageais dans le son! La chaleur était insupportable avec tous ces amplificateurs. J'aurais bien aimé avoir un technicien pour m'aider, mais je devais tout faire moi-même, réparations comprises. En même temps, j'écrivais Contrastes pour l'Orchestre symphonique de Toronto et Seiji Ozawa... Quand j'ai eu terminé Katimavik, tout un groupe du bureau du commissaire général est venu avec le président. Ils se sont assis dans les marches et je les ai submergés! Quatre haut-parleurs de quinze pouces avec des haut-parleurs aigus qui les brassaient avec quelque chose de totalement neuf!

1997: AM.M. - Combien de temps avez-vous pris pour la composer?

0.J. - Beaucoup, beaucoup de temps. Des heures, des semaines, j'étais totalement absorbé. Sans compter que j’écrivais Contrastes en même temps!

AM.M. - Et quelle était votre intention avec Katimavik?

0.J. - Je ne sais pas... C'était une première... Je voulais... j’allais presque dire que j'espérais que le public allait accepter la pièce. Je n'avais jamais fait cela de ma vie et c'était la première pièce entièrement électronique produite au Canada. Il n'y a aucun son concret. Tout d'abord, il fallait s'adapter à un immense environnement. Et puis je voulais surprendre les gens. Il y avait un aspect oriental à la musique, mais, croyez-moi, cela a été purement accidentel: j'avais utilisé une invention de Kenneth Albert et de moi qui créait une série de sous-harmoniques, une série mineure, que j'ai exploitée tout au long de la pièce. Si vous la jouez assez vite, le son devient semblable à celui d'un instrument à anche double, très proche d'une sonorité indienne. J'ai aussi joué avec le facteur vent: les quatre doubles sources sonores produisent chacune un son différent et, quand il y avait un fort vent, cela créait un effet Doppler. J'étais heureux de l'existence naturelle de cet effet, car quant au reste, je combattais plutôt la nature en créant électroniquement ces sousharmoniques. 
2009: AM.M. - Étiez-vous content du résultat?

0.J. - Je ne sais pas si je peux répondre à cette question... J'étais satisfait du résultat, du moins assez satisfait pour le présenter au public. Dans ce cas, le public, cela voulait dire de neuf heures du matin à onze heures du soir, constamment, pour au moins six mois! Je ne peux dire si c'était bien. C'était certainement différent de tout ce qui avait été fait avant: imaginez, quatre pistes! En autant que je sache, il n'y avait pas eu de quatre pistes au Canada, bien qu'il y en ait sûrement eu déjà à l'Université Columbia.

2009: AM.M. - Après cette pièce, et malgré un intérêt constant pour l'électronique, vous ne vous êtes pas spécialisé dans ce genre. J'ai l'impression que pour vous l'électroacoustique est un peu une voie parallèle. J'ai lu dans l'ébauche de vos mémoires ${ }^{14}$ des opinions assez tranchées sur la musique électronique, la considérant moins importante. Vous dites même qu'un compositeur doit être extrêmement prudent en s'en servant. Ai-je bien lu?

$1983^{15}$ : 0.J. - La musique électronique n'existe pas pour moi. Seule la musique par des moyens électroniques peut exister. Aujourd'hui, vous pouvez obtenir de nouveaux sons provenant d'instruments électroniques, c'est vrai. Cela peut même être possible avec d'autres sortes d'instruments. Peut-être bien que oui, peut-être bien que non. Mais moi, j'attends toujours un grand compositeur de musique électronique. Et je ne considère pas Gesang der Jünglinge de Stockhausen comme une pièce électronique. C'est une pièce manipulée, une voix de jeune garçon manipulée. Ça, c'est une bonne pièce. Après ça, je n’ai pas entendu grand-chose de bon!

1997: 0.J. - Je crois encore que même aujourd'hui, avec les avancées technologiques des appareils et des synthétiseurs, il n'y a pas de Beethoven parmi nous. Honnêtement, rien de vraiment valable n'est apparu en musique électronique. Nous adorons toujours les efforts de Varèse avec Déserts ou de Stockhausen avec Gesange der Jünglinge, - mais c'était il y a 40 ans... - et rien n'est apparu depuis! Aucun compositeur de musique électronique n’a surpassé Varèse ou Stockhausen! Pour ma part, je n'ai jamais voulu me reposer uniquement sur la musique électronique même si elle était disponible. Je crois que je suis un des rares compositeurs à écrire tant de la musique électroacoustique que de la musique instrumentale. L'électronique est toujours là, je me suis construit un studio complet au fil des ans ${ }^{16}$, il est très à jour, très facile d'utilisation, et pourtant j'écris pour violoncelle solo et pour d'autres formations qui me plaisent plus. Pourquoi? Je ne sais pas. Peut-être est-ce un peu trop facile?
14. Fonds Otto-Joachim, Bibliothèque et Archives Canada, MUS 270 /A, 6.

15. Tiré de la transcription d'une entrevue de Hugh Davidson avec Otto Joachim pour l'Anthologie de la musique canadienne, vol. 14: 1983; RCl 5-ACM14, Fonds Otto-Joachim, Mus 270/ B, 11, traduction de l'auteure.

16. Outre l'équipement déjà mentionné dans le texte, le studio comprend aussi des modulateurs en anneau et des oscillateurs à gradins. Lorsqu'en 1969, Otto Joachim est devenu agent commercial canadien de EMS Synthesizers (Angleterre), son studio s'est enrichi de nombreux éléments de ce fabricant, comme le Synthi AKS, le Pitch to Voltage Converter, le Random Generator ou le Vocoder. On y retrouve aussi un Waldrof Pulse, un Yamaha DX7, un Yamaha FB-01 et un Korg EX-8000. 
1997: AM.M. - Comment cela peut-il être trop facile?

0.J. - Le problème avec la musique électronique aujourd'hui est qu'il y a tant d'inventions disponibles que la paresse guette le compositeur qui ne tente plus d'inventer ses propres sons, car ils sont déjà fabriqués. Il n'y a plus de raison pour lui de se perfectionner avec la technique de composition, avec la structure, avec son architecture. Il devient un opportuniste de la composition. Avec tous les équipements récents, je pourrais tromper quiconque. On me demande souvent: «Comment vous avez fait ça? Dans ce cas, la seule vraie réponse serait: «J'ai tourné un bouton!» Je peux rendre mes sons immédiatement crédibles, même pour un compositeur, surtout pour un compositeur! Les compositeurs sérieux ont généralement très peur de la musique électronique, car ils ont souvent des étudiants qui, techniquement, sont bien plus avancés qu'eux! Et qui sont peut-être aussi plus aventureux. Je suis une combinaison de vieux et de jeune. Dans mon esprit, je compétitionne avec n’importe quel jeune, parlant de musique bien sûr.

2009: AM.M. - Peut-être en viendra-t-il un exceptionnel dans le prochain siècle?

0.J. - C'est impossible! Parce qu'il n'utilisera toujours que les haut-parleurs! Je ne peux prédire ce qui arrivera et je n'aime pas faire ce genre de prédiction, mais quand je regarde tout ce que les compositeurs de musique électronique ont produit de mieux, avec leurs idiosyncrasies propres, aucun n'a atteint la puissance de la musique du passé.

2009: AM.M. - Mais quelles sont exactement ces limitations?

0.J. - La limite des haut-parleurs est que vous ne voyez personne devant vous qui fait la musique! Depuis le début, je le répète, il n'y aura jamais de Beethoven électronique. Ce que Beethoven a dit dans le champ tonal est déjà la perfection. Ce que Schoenberg, Webern et Berg ont fait au chapitre du dodécaphonisme atteint la perfection. Ce que Hindemith ou Milhaud ont dit dans leurs champs propres - quel qu'en soit le nom - est aussi la perfection. Nous sommes confinés en musique sérielle ou tonale parce qu'on ne peut aller plus bas qu'une corde de contrebasse ou une note de tuba, qu'on ne peut aller plus haut qu'un piccolo. On peut faire toutes sortes d'autres choses avec la musique électronique! Nous pouvons inventer un monde de merveilles, mais ce merveilleux a-t-il autant de valeur que ce qui a précédé? J'en doute. 
LISTE DES EUVRES D'OTTO JOACHIM COMPORTANT

DE L'ÉLECTROACOUSTIQUE

- Katimavik, 1967 , bande quatre pistes

- Illumination II, 1969, ensemble, bande quatre pistes et projecteurs ${ }^{17}$

- 5.9, 1971, bande quatre pistes

- 6 1/2, 1971, bande quatre pistes

- Mankind, 1972, 4 récitants, 4 synthétiseurs, orgue, timbales, encens, diapositives et projecteurs

- Urauffuihrung, 1976-1977, guitare solo, ensemble et sons électroniques en direct

- Stimulus à Goad, 1977, guitare et synthétiseur

- Seven Electronic Sketches, 1984, bande quatre pistes

- Mobile fûr Johann Sebastian Bach, 1980-1985, ensemble et bande

- Elektroakustische Momente, 1999, album de 36 pièces électroacoustiques

- Three Electronic Sketches, 2000, bande quatre pistes

\section{DISCOGRAPHIE MONOGRAPHIQUE}

JOACHIM, Otto (1983), Anthologie de la musique canadienne, vol. 14 (RCI ACM14): Music for violin and viola, Sonata for cello and piano, L'Éclosion, Concertante $n^{\circ} 1$, Quatuor à cordes $n^{\circ} 1,12$ Twelve-tone pieces for children, Psalm, Fantasia, Nonet, Dialogue, 5.9, Divertimento, Katimavik, Six pieces for guitar, Illumination II, Stimulus à Goad, Night Music, Intermezzi, Tribute to St-Romanus; Otto Joachim, John Newmark, William Albright, Louis Charbonneau, Davis Joachim, Harvey Seigel, Jacques Beaudry, Walter Joachim, Hyman Bress, Kenneth Gilbert, Robert Verebes, Suzanne Blondin, Tristan Nguyen, Quintette à vent du Québec.

JOACHIM, Otto (2000), «Hommage à Otto Joachim», Radio-Canada, les Productions Riche Lieu: Stacheldraht, Quatuor à cordes, Métamorphoses, Paean, Illumination II; Ensemble de la SMCQ (dir. Walter Boudreau), Quatuor Molinari, Orchestre Métropolitain (dir. Joseph Rescigno), Guy Fouquet, Ensemble instrumental de Radio-Canada (dir. Otto Joachim). JOACHIM, Otto (2000), Elektroakustische Momente, Amtech.

\section{B I B L I O GRAPHIE}

Fonds Otto-Joachim, Bibliothèque et Archives Canada, mus 270. 